\title{
Pyruvate and Ethanol as Electron Donors for Nitrite Reduction by Escherichia coli K12
}

\author{
By N. R. POPE† AND J. A. COLE* \\ Department of Biochemistry, University of Birmingham, Birmingham B15 2TT, UK
}

(Received 14 November 1983; revised 16 January 1984)

\begin{abstract}
Pyruvate and ethanol were both effective electron donors for nitrite reduction by Escherichia coli K12. The pyruvate-dependent rate decreased by approximately $50 \%$ when either a cysG mutation, which results in loss of NADH-dependent nitrite reductase activity (EC 1 6.6.4), or a chl mutation, which results in loss of the formate-nitrite oxidoreductase activity, was introduced into the prototrophic parental strain CGSC4315. A double mutant deficient in both of these previously described activities retained only $2 \%$ of the rate of nitrite reduction of the parental strain after growth on glucose or $5 \%$ after growth on pyruvate. We conclude that any third pathway for nitrite reduction contributes little to the in vivo rate of nitrite reduction by wild-type strains.
\end{abstract}

\section{INTRODUCTION}

Anaerobic, wild-type cultures of Escherichia coli $\mathrm{K} 12$ are able to reduce nitrite using formate (Abou-Jaoudé et al., 1977, 1979) or NADH (Kemp \& Atkinson, 1966; Cole et al., 1974; Jackson et al., 1981) as reductant; the enzymes which catalyse these two reactions are independent (Pope \& Cole, 1982). The formate-nitrite oxidoreductase complex is membrane-bound and conserves energy by oxidative phosphorylation during nitrite reduction (Pope \& Cole, 1982). In contrast, NADH-nitrite reductase (EC 1.6.6.4) is a soluble cytoplasmic enzyme (Cole \& Wimpenny, 1968). In intact bacteria glucose can also act as a reductant for nitrite.

Pascal et al. (1981) reported that pyruvate was able to donate electrons for nitrite reduction in strains of $E$. coli that were deficient in NADH-nitrite reductase activity and unable to produce formate from pyruvate due to a lack of pyruvate formate-lyase activity (acetyl-CoA : formate $C$ acetyltransferase; EC 2.3.1.54). An aceE mutant was unable to use pyruvate to reduce nitrite: this suggested to Pascal et al. (1981) that the pyruvate dehydrogenase complex might interact directly with a third major nitrite reductase, independent of the previously described nitrite reductases. However, the demonstration that a strain of E. coli deficient in both the NADH- and formate-dependent nitrite reductase activities retained less than 2 to $5 \%$ of the activity in the parental strain with glucose as reductant appeared inconsistent with this suggestion (Pope \& Cole, 1982). We now report the use of the previously described set of four isogenic strains to investigate the enzymes involved in nitrite reduction by pyruvate.

\section{METHODS}

Bacterial strains, growth conditions and the preparation of washed cell suspensions have all been described previously (Pope \& Cole, 1982). Soluble extracts were prepared using washed cells that had been resuspended in $60 \mathrm{~mm}$-Tris $/ \mathrm{HCl}, \mathrm{pH} 8 \cdot 8$. This suspension was passed through a French pressure cell at a pressure of approx. $60 \mathrm{MPa}$ and the resulting extract was centrifuged at $260000 \mathrm{~g}$ for $1 \mathrm{~h}$ (Newman \& Cole, 1978). After centrifugation the supernatant ('soluble extract') was removed and used to determine alcohol dehydrogenase activity and cytochrome $c_{552}$ concentration.

† Present address: Biotechnology Department, PA Technology, Melbourn, Royston, Herts. SG8 6DP, UK. 
The rate of nitrite reduction by cell suspensions was determined using the discontinuous assay of Pope \& Cole (1982): pyruvate and ethanol replaced formate as electron donor. Alcohol dehydrogenase activity was determined spectrophotometrically by the method of Kersters \& De Ley (1966).

The cytochrome $c_{552}$ concentration in soluble extracts was determined by measuring the dithionite-reduced versus nitrite-oxidized difference spectrum. Samples of soluble extract $(1 \mathrm{ml})$ were diluted twofold in $50 \mathrm{~mm}$ -

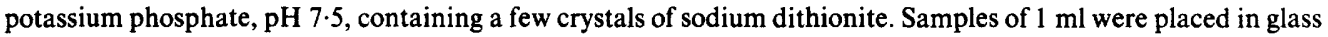
cuvettes and a reduced versus reduced baseline spectrum was recorded. A few crystals of sodium nitrite were then added to the reference cuvette, and the reduced versus nitrite-oxidized spectrum was recorded over the wavelength range 500 to $600 \mathrm{~nm}$. Cytochrome $c_{552}$ concentration was calculated from peak height and a value of $\varepsilon_{552}$ for the difference spectra of $24100 \mathrm{M}^{-1} \mathrm{~cm}^{-1}$ (Fujita, 1966).

\section{RESULTS}

\section{Nitrite reduction by pyruvate}

Whole-cell suspensions of strain CGSC 4315 could use pyruvate to reduce nitrite (Fig. 1). The rate of this reaction was decreased by approximately $50 \%$ in derivatives of this strain deficient in NADH- or formate-dependent nitrite reduction (strains carrying the cysG3102 or chl-401 mutation). Strain CB403, which carries both the cysG3102 and chl-401 mutations, retained only 0 to $5 \%$ of the activity of the parental strain. These results suggest that both the NADH- and formate-dependent pathways for nitrite reduction are involved in electron transfer from pyruvate.

From Fig. 1 it can be seen that the rate of nitrite reduction by pyruvate accelerated during the experiment. This was also true for strains CB401 and CB402, and nitrite utilization by strain CB403 only became apparent towards the end of the assays (data not presented). Because of this the values given in Table 1 are the rates of nitrite reduction by pyruvate that were obtained after a 25 min incubation with pyruvate and ethanol.

Possible explanations for the acceleration of the pyruvate-dependent rates of nitrite reduction were investigated further with strain CGSC4315 (Fig. 2). Cell suspensions were pre-incubated with pyruvate for $30 \mathrm{~min}$ before the assays were initiated by the addition of nitrite. The rate obtained, $40.3 \mathrm{nmol}$ nitrite reduced $\min ^{-1}(\mathrm{mg} \text { dry wt })^{-1}$ was higher than had previously been obtained (Table 1), and did not accelerate. The presence of chloramphenicol $\left(200 \mu \mathrm{g} \mathrm{ml}^{-1}\right)$ during an assay in which pyruvate had been excluded from the pre-incubation resulted in a constant rate of $9 \cdot 1 \mathrm{nmol}$ nitrite reduced $\min ^{-1}(\mathrm{mg} \text { dry wt })^{-1}$. This was approximately equal to the initial rate obtained without pre-incubation (Fig. 2). As the pyruvate-dependent rate of nitrite reduction was constant after the 30 min pre-incubation with pyruvate, it was concluded that this period was sufficient for full activation. Furthermore, because chloramphenicol can prevent this activation, protein synthesis was implicated in this process.

In order to confirm the effect of chloramphenicol on the activation, rates were measured with bacteria that had been pre-incubated with pyruvate and chloramphenicol. A biphasic response was obtained (Fig. 2). Both sections of the time course were linear; the primary rate was 25.4 and the secondary rate was $12.3 \mathrm{nmol}$ nitrite reduced $\min ^{-1}(\mathrm{mg} \text { dry } \mathrm{wt})^{-1}$. The secondary rate was similar to that obtained using chloramphenicol without pre-incubation with pyruvate (see above), and was approximately half of the primary rate.

The above rates are the means of values obtained during three independent sets of experiments. Variation of the individual values between experiments was less than $10 \%$, except for the primary rate of the biphasic response where $30 \%$ variation was obtained.

The detection of residual nitrite reduction by glucose or pyruvate in strain CB403 suggested that a third nitrite reductase might be present. Possibly this activity could be induced by pyruvate. To investigate this, rates of nitrite reduction by strains CGSC 4315 and CB403 were measured after growth in medium in which glucose was replaced with $0.4 \%(\mathrm{w} / \mathrm{v})$ sodium pyruvate. The rate of nitrite reduction by pyruvate by the parental strain was constant, $27.0 \mathrm{nmol}$ nitrite reduced $\min ^{-1}(\mathrm{mg} \text { dry } \mathrm{wt})^{-1}$, slightly less than the maximum value obtained previously after pre-incubation of glucose-grown cells with pyruvate. This confirms that the 30 min pre-incubation of glucose-grown cells with pyruvate resulted in the maximal expression of activity. The double mutant CB403 was devoid of NADH- or formate-dependent activities 


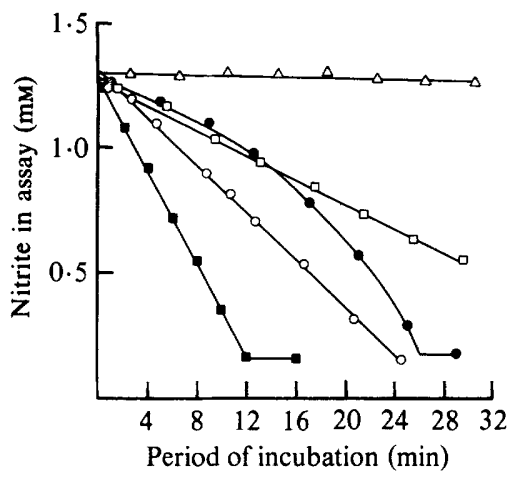

Fig. 1

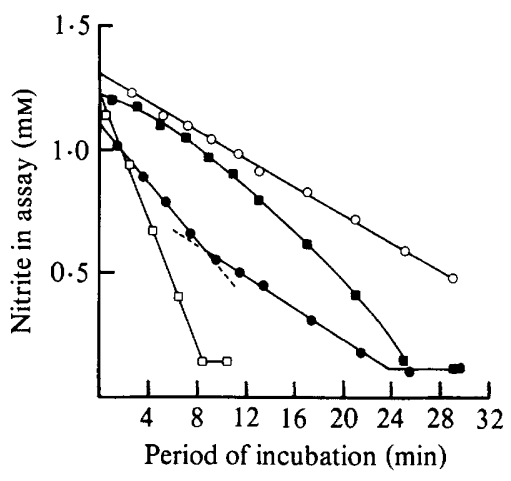

Fig. 2

Fig. 1. Nitrite reduction by whole cell suspensions of strain CGSC4315 was measured in the absence of an added reductant $(\triangle)$, and in the presence of glucose $(\square)$, pyruvate $(\Theta)$, formate $(O)$ or ethanol $(\square)$, all at $50 \mathrm{~mm}$. Cell densities were $1.4 \mathrm{mg}^{\mathrm{dry}} \mathrm{wt} \mathrm{ml}^{-1}$ in the presence of glucose $(\square)$ and $2.8 \mathrm{mg}$ dry $\mathrm{wt} \mathrm{ml}^{-1}$ in all other assays. Similar results were obtained in a further three independent experiments.

Fig. 2. Nitrite reduction by pyruvate was assayed with $(\square)$ or without $(\square) 30$ min pre-incubation of the cell suspension with pyruvate. Assays were started by the addition of nitrite. Also shown are the effects of chloramphenicol $\left(200 \mu \mathrm{g} \mathrm{ml}^{-1}\right)$ in the absence of pre-incubation $(\mathrm{O})$ and when present during the pre-incubation (O). In each assay the concentration of the cell suspension was $3 \cdot 3 \mathrm{mg}$ dry wt $\mathrm{ml}^{-1}$. Similar results were obtained in a further two independent sets of experiments.

Table 1. Nitrite reduction by Escherichia coli K12 strains after growth in glucose

Nitrite reduction with washed bacteria [nmol nitrite reduced $\min ^{-1}$ (mg cell dry $\left.w t)^{-1}\right]$

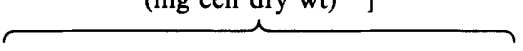

Strain

With With With With
glucose* pyruvate formate* ethanol

CGSC4315
(parental strain)
CB401 chl-401
CB402 cysG3102
CB403 cysG3102
chl-401

$\begin{array}{rccc}66.9 & 18.5 & 46.0 & 9.4 \\ 63.0 & 8.8 & <0.5 & <0.5 \\ 22.6 & 10.6 & 32.0 & 6.6 \\ 1.6 & <0.5-1.0 & <0.5 & <0.5-1.0 \dagger\end{array}$

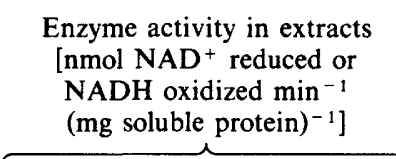

$\begin{array}{rl}56 & 204 \\ 5 & 537 \\ 55 & <1 \\ 21 & <1\end{array}$

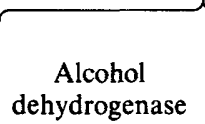

Cytochrome $c_{552}$ [pmol (mg soluble dehydrogenase oxidoreductase* protein $^{-1}$ ]

* Data from Pope \& Cole (1982) included for comparison.

$\dagger$ In four experiments two values of $<0.5$ and two of 1.0 were obtained.

[ $<1$ and $<0.5 \mathrm{nmol}$ nitrite reduced $\min ^{-1}(\mathrm{mg} \text { dry } w \mathrm{t})^{-1}$, respectively] but the pyruvatedependent activity was $1.3 \mathrm{nmol}$ nitrite reduced $\min ^{-1}(\mathrm{mg} \text { dry } \mathrm{wt})^{-1}$. This residual activity was two to three times the rate obtained with glucose-grown cells, was detectable from the beginning of the assays, and did not accelerate. These results are the average of two independent determinations, the variation between experiments being less than $10 \%$.

The above results are consistent with the existence of a third but extremely minor pathway for nitrite reduction which might possibly be the pyruvate-dependent activity proposed by Pascal $e t$ al. (1981). Furthermore, these results appear to demonstrate induction of this activity by pyruvate. 


\section{The reduction of nitrite by ethanol}

Nitrite reduction by ethanol (Fig. 1) has not previously been demonstrated. This substrate was originally tested because it was assumed that nitrite reduction by ethanol would be catalysed entirely by the NADH-dependent nitrite reductase, the NADH being produced from ethanol by alcohol dehydrogenase activity. However, from the ethanol-dependent activity in derivatives of strain CGSC4315 (Table 1) it was obvious that this notion is incorrect because the NADHnitrite reductase-deficient strain CB402 was able to utilize ethanol for nitrite reduction. However, the rate of nitrite reduction in this strain was less than that of the parental strain suggesting that some of the ethanol-dependent activity of the parental strain is due to the NADH-nitrite reductase. The chl-401 lesion either eliminated or severely lowered the ethanoldependent nitrite reductase activity of strains CB401 and CB403, respectively. The total lack of activity in a strain with an active NADH-nitrite reductase is apparently inconsistent with the involvement of this enzyme. However, to ensure that ethanol was being metabolized in these strains, alcohol dehydrogenase activities were measured. The alcohol dehydrogenase activity in strain CB401 was much lower than the activity found in strain CGSC4315 and CB402 (Table 1). The lack of ethanol-dependent nitrite reduction in strain CB401 is therefore probably due to failure to metabolize sufficient ethanol to provide the substrate for nitrite reduction, NADH. An intermediate alcohol dehydrogenase activity was detected in strain CB403, which is devoid of formate- or NADH-dependent nitrite reductase activities. The residual rate of ethanoldependent nitrite reduction in strain CB403 is further evidence for a third, previously undescribed but minor pathway for nitrite reduction.

\section{DISCUSSION}

The rate of nitrite reduction by pyruvate was about $50 \%$ lower in mutants lacking either the formate or the NADH-dependent nitrite reduction pathways than in the isogenic strain and was virtually absent in the double mutant (Table 1). These results are clearly inconsistent with the proposal of Pascal et al. (1981) that nitrite reduction by pyruvate is catalysed by a third pathway involving neither NADH nor formate. Although we have confirmed that formate is an ineffective electron donor for one of the strains, LCB197, on which the conclusions of Pascal et al. (1981) were based, we have detected cytochrome $c_{552}$ and high activities of NADHdependent nitrite reductase in this mutant (unpublished observations). The latter could readily explain why pyruvate is an effective electron donor for nitrite reduction by LCB197. We conclude, therefore, that pyruvate is an effective electron donor for nitrite reduction because it is the precursor of both of the substrates for the two major nitrite reductases of $E$. coli. Formate is formed from pyruvate as the product of the pyruvate formate-lyase activity (Knappe et al., 1974), whereas NADH formation appears to be catalysed by the pyruvate dehydrogenase complex, as stated by Pascal et al. (1981).

The rate of nitrite reduction by pyruvate accelerated during the assays unless chloramphenicol was included. A maximal and constant rate was obtained after 30 min pre-incubation of glucosegrown bacteria with pyruvate in the absence of chloramphenicol, or by using bacteria which had been grown with pyruvate as carbon source. The demonstration that protein synthesis is required to achieve the optimal rate of nitrite reduction by pyruvate indicates that enzyme induction is occurring. The acceleration was observed with all four strains, suggesting it is the pyruvate transport system (Kornberg, 1967) rather than the metabolism of pyruvate to formate or NADH that is being induced. If pyruvate transport is the rate-limiting step in nitrite reduction by pyruvate, increased synthesis of the pyruvate permease would inevitably result in a more rapid production of the substrates for nitrite reduction.

When protein synthesis was inhibited during pre-incubation with pyruvate and nitrite was then added, a biphasic rate of nitrite reduction was detected. The slower, secondary rate was similar to and probably reflects the uninduced rate. The primary rate was more rapid and can be explained as follows. During the pre-incubation with pyruvate and in the absence of nitrite, formate will accumulate. When nitrite is added, reduction occurs at the faster rate until there is insufficient formate to saturate the formate-nitrite oxidoreductase pathway. Eventually the 
reaction rate returns to the uninduced rate determined by the rate of formation of formate and NADH from extra-cellular pyruvate. The kinetics of nitrite reduction by pyruvate are therefore totally compatible with the view that pyruvate is simply a precursor of the substrates for the two previously characterized nitrite reductases.

The relative contribution of the two major pathways for nitrite reduction is strain dependent. Strain CGSC4315 was selected for the present work because the rate of nitrite reduction by formate is approximately $50 \%$ of the glucose-dependent rate, far higher than that of other strains such as AB2847 (20\%), OR56 (14\%) or MC4100 (6\%) (unpublished observations). The significance of data obtained with a strain such as CGSC4315 is greater because differences in rates of nitrite reduction between derivatives lacking one or other pathway and the parental strain are far greater than the experimental errors inherent in the methods used. In contrast to the major role of the formate and NADH-dependent pathways in nitrite reduction by pyruvate, the residual rate of nitrite reduction by strain CB403 is barely significant, though it is conceivable that this would have been higher in derivatives of other strains. Any third pathway for nitrite reduction by pyruvate contributes at most $2 \%$ or $5 \%$ to the rates of nitrite reduction by the parental strain CGSC4315 grown with glucose or pyruvate, respectively.

Recently there have been reports from at least three laboratories of a third pathway for nitrite reduction by fermentative bacteria in which nitrite reduction to nitrous oxide is apparently catalysed by nitrate reductase (Kaspar \& Tiedje, 1981; Smith, 1983; Satoh et al., 1983). As the preferred electron donors for nitrate reduction are formate and $\mathrm{NADH}$, there is no reason to propose that such a gratuitous activity provides a pathway for nitrite reduction by pyruvate which is independent of NADH or formate. Less clear, however, is the significance of the moderately high rate of nitrite reduction by ethanol with strain CB402 which lacks NADHnitrite oxidoreductase activity. As a working hypothesis we suggest that, when nitrite is available, reoxidation of NADH by wild-type bacteria is catalysed mainly by the soluble, NADH-dependent nitrite reductase, but a small proportion is oxidized by the anaerobic respiratory chain which transfers electrons to a terminal nitrite reductase (possibly cytochrome $c_{552}$ which was readily detected in all of the four isogenic strains; Table 1 ). This minor pathway for nitrite reduction could account for the significant rate of nitrite reduction by ethanol with strain CB402 as well as the requirement for an active pyruvate dehydrogenase for nitrite reduction by pyruvate when the formate pathway for nitrite reduction is inactive, as reported by Pascal et al. (1981).

N. R. P. was supported by an NERC Studentship. The authors are grateful to Dr Marc Chippaux for providing strain LCB197 so that the interpretation of earlier results could be reassessed and for helpful discussions.

\section{REFERENCES}

Abou-Jaoudé, A., Chippaux, M., Pascal, M. C. \& CASSE, F. (1977). Formate: a new electron donor for nitrite reduction in Escherichia coli K12. Biochemical and Biophysical Research Communications 78, 579583.

Abou-Jaoudé, A., Chippaux, M. \& Pascal, M. C. (1979). Formate-nitrite reduction in Escherichia coli K12. Physiological study of the system. European Journal of Biochemistry 95, 309-314.

Cole, J. A. \& WimpennY, J. W. T. (1968). Metabolic pathways for nitrate reduction in Escherichia coli. Biochimica et biophysica acta 162, 39-48.

Cole, J. A., Coleman, K. J., Compton, B. E., KavanaGh, B. M. \& KeEvil, C. W. (1974). Nitrite and ammonia assimilation by anaerobic continuous cultures of Escherichia coli. Journal of General Microbiology 85, 11-22.

FujITA, T. (1966). Studies on soluble cytochromes in the Enterobacteriaceae. I. Detection, purification and properties of cytochrome $c_{552}$ in anaerobically grown cells. Journal of Biochemistry 60, 204-215.
JaCkson, R. H., CoRnish-Bowden, A. \& COLE, J. A. (1981). Prosthetic groups of the NADH-dependent nitrite reductase from Escherichia coli K12. Biochemical Journal 193, 861-867.

Kaspar, H. F. \& Tiedje, J. M. (1981). Dissimilatory reduction of nitrate and nitrite in the bovine rumen: nitrous oxide production and effect of acetylene. Applied and Environmental Microbiology 41, 705709.

Kemp, J. D. \& Atkinson, D. E. (1966). Nitrite reductase of Escherichia coli specific for reduced nicotinamide adenine dinucleotide. Journal of Bacteriology 92, 628-634.

KerSTERS, K. \& DE LEY, J. (1966). Soluble NADlinked primary and secondary alcohol dehydrogenases. Methods in Enzymology 9, 347-350.

KNAPPe, J., Blaschkowski, H. P., Grobner, P. \& SchmitT, T. (1974). Pyruvate-formate lyase of Escherichia coli: the acetyl enzyme intermediate. European Journal of Biochemistry 50, 253-263.

KORNBERG, H. L. (1967). Genetic control of the uptake 
of pyruvate by Escherichia coli. Biochimica et biophysica acta 148, 591-592.

Newman, B. M. \& Cole, J. A. (1978). The chromosomal location and pleiotropic effects of mutations of the nirA ${ }^{+}$gene of Escherichia coli K12: the essential role of nir $A^{+}$in nitrite reduction and in other anaerobic redox reactions. Journal of General Microbiology 106, 1-12.

Pascal, M. C., Chippaux, M., Abou-Jaoudé, A., BlaschKowsKi, H. P. \& KNAPPE, J (1981). Mutants of Escherichia coli $\mathrm{K} 12$ with defects in anaerobic pyruvate metabolism. Journal of General Microbiology 124, 35-42.
Pope, N. R. \& Cole, J. A. (1982). Generation of a membrane potential by one of two independent pathways for nitrite reduction by Escherichia coli. Journal of General Microbiology 128, 219-222.

Satoh, T., Ном, S. S. M. \& Shanmugam, K. T. (1983). Production of nitrous oxide from nitrite in Klebsiella pneumoniae: mutants altered in nitrogen metabolism. Journal of Bacteriology 155, 454-458.

SMITH, M. S. (1983). Nitrous oxide production of Escherichia coli is correlated with nitrate reductase activity. Applied and Environmental Microbiology 45, 1545-1547. 